\title{
Optimization of Detection Schemes in BOTDA
}

\author{
Li Zhang ${ }^{1,2}$, Marcelo A. Soto ${ }^{2}$, Zinan Wang ${ }^{1}$, and Luc Thévenaz ${ }^{2}$ \\ ${ }^{1}$ Key Lab of Optical Fiber Sensing \& Communications, University of Electronic Science \&Technology of China, Chengdu, China 611731 \\ ${ }^{2}$ EPFL Swiss Federal Institute of Technology, Institute of Electrical Engineering, SCI STI LT, Station 11, CH-1015 Lausanne, Switzerland \\ Authore-mail address: luc.thevenaz@epfl.ch
}

\begin{abstract}
The impact of different detection schemes on the performance of Brillouin optical timedomain analysis is investigated theoretically and experimentally. The study provides guidance for the optimization of BOTDA sensors depending on the targeted range.

OCIS codes: (060.2310) Fiber optics; (060.2370) Fiber optics sensors; (290.5900) Scattering, stimulated Brillouin
\end{abstract}

\section{Introduction}

Brillouin optical time-domain analysis (BOTDA) has been studied for many years for distributed temperature and strain sensing [1]. The principle is based on the temperature and strain dependence of the Brillouin frequency shift (BFS). In order to retrieve the BFS profile along a given sensing range, the sensor scans the Brillouin gain spectrum (BGS) at each position along the fiber and retrieves the Brillouin gain peak frequency through a fitting procedure [2].

The parameter of uttermost importance that essentially determines the quality of the measurements is the signalto-noise ratio (SNR) [2]. With the aim of enhancing the performance of BOTDA sensors, several advanced techniques have been proposed over the last decade to improve the SNR of the measurements [1-5]. However, in addition to these sophisticated methods, different detection schemes have also been proposed in the literature, such as the use of an optical pre-amplification stage in front of the detector in a direct detection scheme [6], balanced detection [7] and coherent detection [8]. Each of these schemes has demonstrated an improvement in the performance of the sensor; however it still remains unclear which of these methods offers the highest improvement in the SNR.

In this paper the impact of different detection schemes (pre-amplified, balanced and coherent detections) on the SNR of the measurements is investigated for BOTDA sensing. Results show that optical pre-amplification offers the simplest and most efficient method to enhance the SNR in long range sensing, while balanced detection is the optimal solution over short ranges. On the other hand, although coherent detection improves the SNR of very low power signals, no real SNR improvement on BOTDA traces is observed when compared to other detection schemes.

\section{Principle}

As a reminder, the noise $\sigma_{d d}$ in a simple direct detection scheme is essentially given by the variance summation of thermal noise $\sigma_{t h}$ and shot noise $\sigma_{s h}$ :

$$
\sigma_{d d}^{2}=\sigma_{t h}^{2}+\sigma_{s h}^{2}=F_{n} \frac{4 K_{B} T}{R_{L}} \Delta f+2 q\left(s P_{s}+I_{d}\right) \Delta f
$$

where $K_{B}$ is the Boltzmann constant, $T$ is the photodetector temperature, $R_{L}$ is the input noise-generating resistance of the detector, $F_{n}$ is the photodetector noise figure, $\Delta f$ is the photodetector bandwidth, $q$ is the electron charge, $P_{s}$ is the optical power of the signal, $s$ is the responsivity of the detector and $I_{d}$ is the photodetector dark current, which can be normally ignored.

Figure 1 shows the behavior of the SNR as a function of the input power.

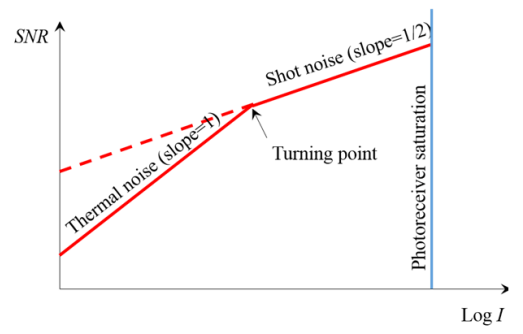

Fig. 1. SNR vs optical power of signal We can see that the SNR is essentially thermal noise-dominated in a low optical power regime. This noise is independent of the input power, so that any increase in the optical power rises the SNR proportionally. On the other hand, at higher optical power shot noise dominates and, since it is power-dependent, any raise in optical power increases the SNR but following a square-root dependence on the input power. In any of these cases the highest SNR is obtained when the output voltage of the detector is near the saturation level (blue line in Fig. 1).

In long range sensing, reaching this optimal condition is actually very difficult due to the low optical power typically reaching the receiver, and therefore the simplest alternative to improve the SNR is to use a pre-amplifier (e.g. an EDFA) in front of the photo-detector. It should be noted that measurements in a BOTDA sensor are mainly made of a strong DC component topped by a small sensor response containing the relevant information [2,9]. Unfortunately the saturation of the electrical detection stage is mostly reached by the non-informative DC component of the signal, imposing a serious limitation to the maximum SNR attainable with direct detection. However, the SNR can be further improved using higher optical powers combined with balanced detection [7]. In this case, a continuous-wave (CW) light can be launched into the second detector input, to compensate the DC component contained in the BOTDA traces, 
thus avoiding saturation of the electrical stages of the receiver. This way, the optical power launched into the photoreceiver can be much higher than the nominal saturation level of the detector, providing a potential increase in SNR.

On the other hand, coherent detection can be used to turn the detection shot noise-limited. This has been one of the first reasons motivating the use of coherent detection in BOTDA sensing [8], and has led researchers to claim a better SNR with respect to direct detection. This however has never been demonstrated strictly under the same experimental conditions and comparing optimized detection schemes. In order to clarify this issue, we should consider that the optical local oscillator (OLO) in this kind of schemes has to be strong enough to make the detection shot noise-limited. A $3 \mathrm{~dB}$ coupler can therefore be used to combine the probe signal reaching the detector with a strong OLO; however, a $2 \times 2$ coupler can also enable coherent balanced detection to raise the optical power launched into the detector and avoid electrical saturation. In this case the beating signal at the output of the detector can be written as $i=2 s \sqrt{P_{S} P_{O L O}} \cos (\omega t+\varphi)$, where $P_{O L O}$ and $P_{S}$ are the optical powers of the OLO and signal, respectively, and $\omega$ is their angular frequency difference. Considering that the dominating noise is the shot noise induced by the OLO, which can be considered uncorrelated for the two inputs, the total noise can be expressed as $\sigma_{s h}^{2}=2 s e P_{O L O} \Delta f$. The signal and noise voltages after passing through a transimpedance gain $R$ and high-pass filtering are:

$$
U=2 R s \sqrt{P_{S} P_{O L O}} \cos (\omega t+\varphi) \quad \sigma_{U}^{2}=2 \operatorname{seR}^{2} P_{O L O} \Delta f
$$

In order to detect the envelope of this beating signal, the electrical squaring of this signal is here considered based on scheme $\mathrm{C}$ in Fig. 2. After passing through an electrical splitter and mixer, the signal and noise read as:

$$
\begin{gathered}
U^{2}=(2 R s)^{2} P_{S} P_{O L O} \cos ^{2}(\omega t+\varphi) \approx \frac{1}{2}(2 R s)^{2} P_{S} P_{O L O} \\
2 U \sigma_{U}=4 R s \sqrt{P_{S} P_{O L O}} R \sqrt{2 s e P_{O L O} \Delta f^{\prime}}=R^{2} s P_{O L O} \sqrt{32 s e P_{S} \Delta f^{\prime}}
\end{gathered}
$$

The bandwidth has been here changed to $\Delta f^{\prime}$ to consider the electrical filtering. In addition, the cosine term of $U$ has been omitted in Eq. (4) since the broadband white noise has equal amplitude at any frequency and this frequency shift (cosine term) does not impact on noise amplitude. So, assuming a shot-noise limit, the SNR can be written as:

$$
S N R_{C d}=\frac{\frac{1}{2}(2 R S)^{2} P_{S} P_{O L O}}{R^{2} S P_{O L O} \sqrt{32 S e P_{S} \Delta f^{\prime}}}=\frac{1}{2} \frac{\sqrt{s P_{S}}}{\sqrt{2 e \Delta f^{\prime}}}
$$

Equation (5) demonstrates that the power of the OLO has no impact on the overall SNR, and coherent detection basically induces a $3 \mathrm{~dB}$ penalty over the SNR obtained by a shot noise-dominated direct detection scheme. More importantly, according to Eq. (5) the SNR here obtained is actually expected to be very small due to the low signal power $P_{S}$ being detected. Therefore, compared to direct detection of a high-power signal (e.g. using pre-amplification), coherent detection does not offer any SNR enhancement in BOTDA sensing.

\section{Experimental setup}

Figure 2 shows the experimental setup used to compare the SNR obtained by different detection schemes. This corresponds to a standard $50 \mathrm{~km}$-long BOTDA sensor with $2 \mathrm{~m}$ spatial resolution. Three detection schemes are implemented (dotted boxes): Scheme A corresponds to direct detection with/without pre-amplifier. Scheme B is a balanced detection, in which a second laser is used to compensate the DC component from the BOTDA signal. Scheme $\mathrm{C}$ uses a heterodyne detection, in which a second laser is utilized as OLO, with an OLO-signal frequency difference of $300 \mathrm{MHz}$. The electrical beating signal goes through a high-pass filter (HPF) and then is split into two branches. By mixing the signals in

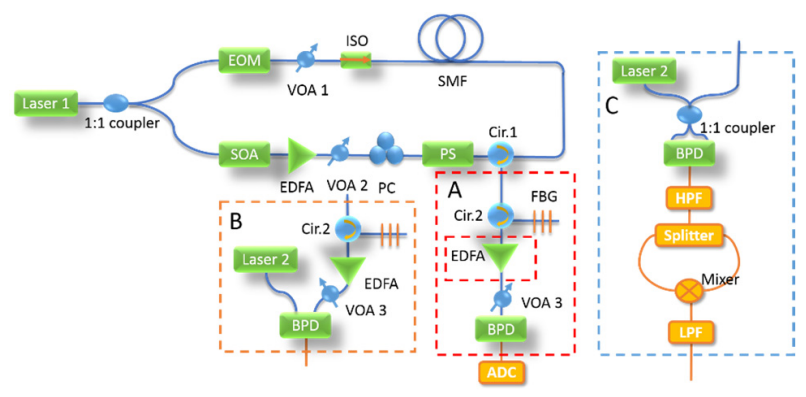

Fig. 2. Experimental setup (EOM: electro-optic modulator, PS: polarization switch, SOA: semiconductor optical amplifier, FBG: fiber Bragg grating, VOA: variable optical attenuator, ISO: optical isolator; Cir: circulator; BPD: balanced photodetector) the two branches and adding a low-pass filter (LPF), the BOTDA trace (envelope of the beating signal) is obtained.

\section{Results and discussions}

The pump-probe frequency offset has been set to the average Brillouin frequency shift (BFS) of the fiber, and several measurements have been consecutively obtained. The SNR is calculated as the mean value over the standard deviation of the measurements at each fiber location. SNR values are compared at the farthest end of the fiber, using a balanced detector of $350 \mathrm{MHz}$ bandwidth. However, the electrical bandwidth of the analog-to-digital (AD) conversion has been set to $100 \mathrm{MHz}$ to suppress the impact of residual components and ensure the same noise bandwidth for all schemes. 
Results are summarized in Table 1. First, a direct detection without pre-amplification is analyzed. In this case an optical power of $-16 \mathrm{dBm}$ is collected by the photo-detector, which shows a thermal noise of $\sigma_{t h}=24.0 \mu \mathrm{V}$ (measured). After measuring the total noise $\left(\sigma_{T}=32.1 \mu \mathrm{V}\right)$, a shot noise of $\sigma_{s h}=21.0 \mu \mathrm{V}$ has been estimated. To improve the SNR, a low-noise EDFA has been placed in the receiver front-end, enabling an optical power raising up to $-4.1 \mathrm{dBm}$ (close to saturation), providing an SNR enhancement from $8.8 \mathrm{~dB}$ up to $10.5 \mathrm{~dB}$. Note that although the signal power is increased by about $12 \mathrm{~dB}$, the SNR turned out to be improved only by $1.7 \mathrm{~dB}$; this is because in addition to the increase of shot noise, the ASE noise is also substantially augmented, thus becoming the dominating source of noise [9]. To further increase the signal in the receiver, a balanced detection is then analyzed. Note that in this case, the same detector is used, while a second laser source is connected to the inverted optical input to subtract the DC level of the BOTDA traces. This configuration increases the total shot noise by a factor $\sqrt{2}$ (for the same input power as before). Using an EDFA and a variable optical attenuator (VOA), the input power on the detector has been raised up to $2.4 \mathrm{dBm}$. Although the amplitude of the measured signal is increased, there is no apparent SNR improvement obtained in this case. This is because the system turns out to be highly dominated by the ASE-signal beating noise [9], and rising the power with a VOA also increases the ASE noise reaching the detector; no SNR improvement could therefore be obtained. It is however believed that a proper optimization of the pump power of the EDFA could lead to an optimized SNR. Finally the SNR of the coherent detection scheme is evaluated. BOTDA traces have been measured in this case with a $350 \mathrm{MHz}$ detection and also repeated with an $800 \mathrm{MHz}$ detector, both cases limited to an electrical bandwidth of $100 \mathrm{MHz}$ in the AD conversion to keep the same detection bandwidth as in the other schemes. Using an OLO power of $5.7 \mathrm{dBm}$ to maximize the output voltage from the detector, an SNR of only $5.8 \mathrm{~dB}$ could be obtained in the best case, being $4.7 \mathrm{~dB}$ below the SNR obtained using a pre-amplification. This SNR is actually slightly lower than the expected SNR since even with a very strong OLO the system could not be fully dominated by shot-noise, resulting in comparable thermal and shot noise levels. This prevents the SNR from reaching the expected optimal value.

Table 1. Experimental results. Values of SNR and noise standard deviation corresponding to 400 averages.

\begin{tabular}{|l|c|c|c|c|c|}
\hline \multirow{2}{*}{ Detection Scheme } & \multicolumn{3}{|c|}{ Measured } & \multicolumn{2}{c|}{ Estimated } \\
\cline { 2 - 6 } & $\begin{array}{c}\text { SNR } \\
(\mathbf{d B})\end{array}$ & $\begin{array}{c}\text { Total noise } \\
(\mu \mathbf{V})\end{array}$ & $\begin{array}{c}\text { Thermal noise } \\
(\mu \mathbf{V})\end{array}$ & $\begin{array}{c}\text { Shot noise } \\
(\mu \mathbf{V})\end{array}$ & $\begin{array}{c}\text { ASE-sig noise } \\
(\mu \mathbf{V})\end{array}$ \\
\hline Direct detection $\left(P_{S}:-16 \mathrm{dBm}\right)$ & $\mathbf{8 . 8 0}$ & 32.1 & $\mathbf{2 4 . 0}$ & 21.2 & - \\
\hline Direct detection + EDFA $\left(P_{S}:-4.1 \mathrm{dBm}\right)$ & $\mathbf{1 0 . 5 4}$ & 267.0 & 24.0 & 83.3 & $\mathbf{2 5 5 . 7}$ \\
\hline Balanced detection + EDFA $\left(P_{S}: 2.4 \mathrm{dBm}\right)$ & $\mathbf{1 0 . 5 3}$ & 1410.5 & 24.0 & 249.1 & $\mathbf{1 3 7 7 . 5}$ \\
\hline Coherent detection $\left(P_{S}:-16 \mathrm{dBm}, P_{O L O}: 5.7 \mathrm{dBm}\right)$ & $\mathbf{5 . 8 4}$ & 15.6 & 10.9 & $\mathbf{1 1 . 1}$ & - \\
\hline
\end{tabular}

\section{Conclusions}

SNRs with different detection schemes in BOTDA have been measured and analyzed. Results suggest that for a weak signal (e.g. in long distance sensing) one can use pre-amplification to raise the signal power just below the saturation level of the detector. For further SNR improvement, an idle CW light can be introduced using a balanced detection to avoid saturation using larger signals. On the other hand, if the signal power is too high (e.g. in short distance sensing), balanced detection can be implemented to avoid saturating the detector, which is a better choice than decreasing the signal power using an optical attenuator since this will certainly impair the SNR. Coherent detection can improve the SNR for a given low signal power, however, the improvement is far from competing with a well-designed preamplification. In long distance BOTDA sensing, pre-amplification is preferred for a better SNR as a simple and effective method. These results can be used as a simple guideline for the optimization of detection schemes in BOTDA.

This work is supported by NSFC (61290312, and 41527805), FRFFTCU (ZYGX2015J008), PCSIRT (IRT1218), the 111 project (B14039). M. A. Soto and L. Thévenaz acknowledge the support from the Swiss Commission for Technology and Innovation (Project 18337.2 PFNM-NM).

\section{References}

[1] A. Motil, et al., "State of the art of Brillouin fiber-optic distributed sensing," Optics \& Laser Technology 78, 81-103 (2016).

[2] M. A. Soto and L. Thévenaz, "Modeling and evaluating the performance of Brillouin distributed optical fiber sensors," Opt. Express 21(25), 31347-31366 (2013).

[3] X. H. Jia, et al., "Experimental demonstration on 2.5-m spatial resolution and $1{ }^{\circ} \mathrm{C}$ temperature uncertainty over long-distance BOTDA with combined Raman amplification and optical pulse coding," IEEE Photonics Technol. Lett. 23(7), 435-437 (2011).

[4] M. A. Soto, J. A. Ramírez, and L. Thévenaz, "Intensifying the response of distributed optical fibre sensors using 2D and 3D image restoration," Nat. Commun. 7, 10870 (2016).

[5] X. H. Jia, et al., "Hybrid distributed Raman amplification combining random fiber laser based 2nd-order and low-noise LD based 1st-order pumping," Opt. Express 21(21), 24611-24619 (2013).

[6] S. Diaz, et al. "A high-performance optical time-domain Brillouin distributed fiber sensor," IEEE Sens. J. 8(7), 1268-1272 (2008).

[7] A. Domínguez-López, et al, "Signal-to-noise ratio improvement in BOTDA using balanced detection," Phot. Tech. Lett. 26(4), 338-341 (2014)

[8] A. Zornoza, M. Sagues, and A. Loayssa, "Self-heterodyne detection for SNR Improvement and Distributed phase shift measurements in BOTDA," J. Lightwave Technol. 30(8), 1066-1072 (2012).

[9] M. A. Soto and L. Thévenaz, "Towards 1000000 resolved points in a distributed optical fibre sensor," Proc. SPIE 9157, 9157C3 (2014). 